\title{
DESENVOLVIMENTO E PADRONIZAÇÃO DE UMA ATIVIDADE LÚDICA PARA O ENSINO DOS CONCEITOS PERTINENTES A SISTEMAS DE PRODUÇÃO
}

\author{
Murilo Pignaton Firme Christo - murilopfc@gmail.com \\ Instituto Federal do Espírito Santo \\ 29150-410 - Cariacica - ES \\ Lúcio Gomes Fontana - luciogfontana@gmail.com \\ Instituto Federal do Espírito Santo \\ 29150-410 - Cariacica - ES \\ Kaylla Lage Jordão - kayllalage@gmail.com \\ Instituto Federal do Espírito Santo \\ 29150-410 - Cariacica - ES \\ Cintia Tavares do Carmo - cintiata@ifes.edu.br \\ Instituto Federal do Espírito Santo \\ 29150-410 - Cariacica - ES
}

Daniela da Gama e Silva Volpe Moreira de Moraes - daniela.moraes@ifes.edu.br Instituto Federal do Espírito Santo

29150-410 - Cariacica - ES

Resumo: Este trabalho consiste no desenvolvimento e padronização de uma atividade lúdica de ensino que segue os princípios norteadores da aprendizagem ativa, com o intuito de contribuir para a melhoria do ensino de conceitos de sistemas de produção do curso de Engenharia de Produção de uma instituição de ensino superior. Além disso, permite que os alunos desenvolvam competências para lidar e resolver problemas encontrados ao longo da vida profissional. O trabalho classifica-se como pesquisa empírica descritiva e, através dos resultados obtidos, conclui-se que a padronização da atividade por meio da utilização de procedimentos operacionais padrões (POPs) é fundamental, pois garante que a atividade será realizada de forma correta. Além disso, assegura a eficácia dos métodos de ensino utilizados, facilita e agiliza o preparo das aulas pelo professor e, consequentemente, economiza tempo de planejamento.

Palavras-chave: Educação em engenharia. Aprendizagem ativa. Padronização de atividades. 


\section{INTRODUÇÃO}

A necessidade de uma formação profissional que acompanhe o ritmo acelerado de transformação do mercado pressiona os sistemas de ensino e faz com que busquem métodos alternativos cada vez mais dinâmicos e lúdicos. Tais métodos de ensino precisam ser capazes de desenvolver tanto competências técnicas quanto habilidades gerenciais, senso crítico e capacidade de trabalhar em grupo (RESENDE et al., 2018; BARBOSA; MOURA, 2014).

Rezende et al. (2018) afirmam que um dos principais entraves que a educação vem enfrentando na didática de ensino é o foco no processo de ensino, ao invés do foco no processo de aprendizagem. No modelo tradicional de ensino, o processo é concentrado na figura do professor e consiste apenas na transmissão da informação. A participação do aluno é quase nula, sendo limitada a apenas tirar dúvidas sobre a informação que foi transmitida, o que pode impedir o desenvolvimento do senso crítico com plenitude.

Segundo Villas-Boas et al. (2011), atualmente os métodos alternativos mais utilizados para sanar a necessidade de sistemas de ensinos mais dinâmicos são as metodologias ativas de aprendizagem como, por exemplo, aprendizagem baseada em problemas, simulação e aprendizagem por projeto. Tais métodos se diferem do modelo tradicional de ensino, pois o processo de aprendizagem passa a ser focado no aluno, que atua diretamente na resolução dos problemas. O professor passa a ser um facilitador, sendo responsável por aplicar o método da melhor forma.

Desse modo, o presente estudo se propõe a apresentar o desenvolvimento e padronização de uma atividade lúdica que segue os princípios norteadores da aprendizagem ativa, com o intuito de possibilitar os alunos do curso de Engenharia de Produção a desenvolverem competências que os capacitem a lidar com problemas encontrados ao longo da vida profissional e resolvê-los.

Este artigo foi resultado de um projeto de pesquisa realizado no Instituto Federal do Espírito Santo, com o suporte do Centro de Excelência em Manufatura, local destinado para o desenvolvimento e aplicação de atividades usando a metodologia ativa no curso de Engenharia de Produção.

\section{EDUCAÇÃO EM ENGENHARIA}

A alta competitividade entre as empresas aliada ao desenvolvimento tecnológico e as rápidas mudanças ocorridas no mundo empresarial, tem demandado que os engenheiros sejam não só capazes de realizar atividades já pré-estabelecidas como características da área, mas também desenvolver tarefas ligadas à gestão de pessoas. Isso exige do profissional a habilidade de transmitir e receber informação, bem como a expertise em lidar com problemas inesperados e resolvê-los (HEINIG, 2015).

Segundo parecer homologado pelo Conselho Nacional de Educação (CNE, 2019), a inserção curricular, deve estar compromissada com a formação de competências e depende da união entre a instituição de ensino e as atividades por ela lecionadas, uma vez que o desenvolvimento do ensino-aprendizagem se dá através de situações práticas que englobam a reflexão e a teorização dos conteúdos. Para tanto, a organização curricular deve adotar estratégias de ensino que integrem e explorem os conteúdos a partir de situações-problema reais ou simulados da prática profissional.

O parecer ressalta ainda que a formação em Engenharia deve ser vista, principalmente, como um processo que possibilite alcançar um resultado satisfatório que seja tecnicamente viável para o usuário final através da utilização do conhecimento técnico da matemática, das 
ciências e das ciências da engenharia. Para que a estrutura curricular atenda tais necessidades, utilizam-se metodologias mais modernas e adequadas à nova realidade global, que estimulam o aluno a buscar fontes diversas de conteúdo e dão ao professor o papel de tutor e mediador de tal processo de aprendizagem (CNE,2019).

Desta forma, o Conselho Nacional de Educação (CNE,2019) destaca que o importante é que tais medidas devem dar sentido, dinamismo e autonomia por meio do engajamento do aluno nas realizações das atividades práticas, desde o primeiro ano do curso. Todas essas medidas têm por finalidade melhorar o ensino e combater a evasão escolar e, para isso, sugere-se a utilização do aprendizado baseado em metodologias ativas ou a resolução de problemas concretos em atividades, instrumentos estes que exigem conhecimentos interdisciplinares.

\section{APRENDIZAGEM ATIVA}

Diversas são as interpretações, de acordo com cada autor, do significado do termo aprendizagem ativa, contudo, Prince (2004) ressalta que é possível fazer uma síntese das definições aceitas pela grande maioria. Define que aprendizagem ativa pode ser considerada como qualquer método que instiga o aluno a ser um participante ativo no processo de aprendizagem. Normalmente, ligadas às atividades de aprendizagem que o envolva e o faça pensar sobre o que está fazendo. Além disso Farias, Martin e Cristo (2015) e Limberger (2013) destacam que o professor deixa de ser o ponto focal da aula e passa a desempenhar o papel de facilitador da aprendizagem, enquanto o aluno passa a construir o seu próprio conhecimento a partir dos recursos disponíveis.

A aprendizagem baseada em problemas tem se destacado no âmbito pedagógico no que se refere ao nível de assimilação e aprendizagem dos conteúdos pelos estudantes, e vem se tornando uma ferramenta importante para a formação de engenheiros. Souza (2015) afirma que com esse método os alunos apresentam os resultados e dominam os conteúdos com mais segurança, visto que estes são obtidos pelos próprios alunos no processo de investigação e de reflexão, desenvolvem as habilidades de formulação de questões e análise crítica de todo o sistema, a fim de solucionarem o problema proposto. Para isso, o indivíduo coleta informações e analisa se elas realmente são importantes para a resolução do problema, além de ser instigado a cooperar, trabalhar em grupo e lidar com todas as situações que vão surgindo ao longo da atividade.

A aprendizagem ativa tem sido um tópico de discussão de ensino em engenharia que vem crescendo cada vez mais em relevância. No Gráfico 1 é possível constatar o aumento do número de artigos publicados sobre o tema no COBENGE. 
Gráfico 1: Artigos Sobre Aprendizagem Ativa Publicados no COBENGE

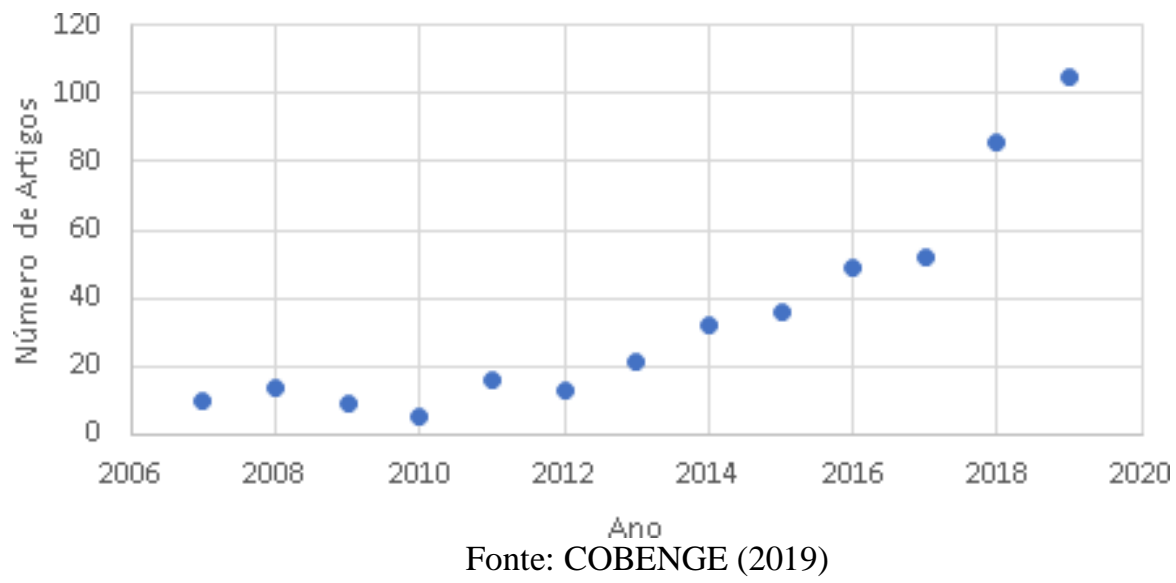

A não participação dos alunos na sala de aula dificulta muito o processo ensinoaprendizagem e diversas vezes está relacionada a falta de motivação. Contudo, várias são as ferramentas que podem ser utilizadas para despertar seu interesse. Costa $(2012$, p.5) cita que "a exploração do aspecto lúdico, pode se tornar uma técnica facilitadora na elaboração de conceitos, no reforço de conteúdo, na sociabilidade entre os alunos, no desenvolvimento da criatividade", e pode muito bem ser trabalhada por meio de jogos interativos, que desenvolvam o raciocínio e simulem situações da realidade.

De acordo com Massa (2016), a combinação da ludicidade com a aprendizagem é um caminho pelo qual se tem a transformação da educação e da construção do conhecimento. É através da vivência da ludicidade, da experiência do lúdico, que o indivíduo se constitui, possibilitando a este a visualização do mundo real através de atividades que utilizem contextos ficcionais.

\section{SISTEMATIZAÇÃO E PADRONIZAÇÃO DE ATIVIDADES}

Segundo Campos (2014), a padronização é uma das principais ferramentas gerenciais, sendo considerada na qualidade total a base para a rotina. Lima e Souza (2015) demonstram que um grande diferencial competitivo perante as organizações atuais é a padronização das atividades por meio de procedimentos operacionais (POP's), que são documentos que explicam detalhadamente a realização das atividades, de modo a criar um padrão que deve ser seguido. Padronizar vai muito além de definir qual é a atividade, quando e onde ela será realizada. É também possibilitar que um indivíduo consiga realizar uma tarefa ou atividade, mesmo não estando familiarizado com ela, de forma que esteja dentro dos padrões definidos como aceitáveis. Ou seja, fazer com que todos entendam o processo de forma clara e objetiva para, assim, realizá-lo de forma acurada (RIZZETTI, 2015).

De acordo com Ribeiro Neto, Tavares e Hoffmann (2013), a padronização de procedimentos possibilita diversas vantagens como o treinamento adequado de colaboradores, a consistência das ações, a garantia da repetibilidade das atividades, além de auxiliar na geração de evidências de que resultados foram alcançados. Padronizar uma atividade lúdica, no formato de um jogo ou simulação, pode facilitar o aprendizado dos alunos e, agilizar o trabalho docente durante o planejamento e execução de uma aula com foco na aprendizagem ativa.

Segundo Pazin Filho e Scarpelini (2007, p.162), simulação consiste em "uma técnica de ensino que se fundamenta em princípios do ensino baseado em tarefas e se utiliza da reprodução parcial ou total destas tarefas em um modelo artificial, conceituado como simulador". Os 
autores salientam ainda que sua aplicação é relacionada, de forma geral, às atividades práticas que envolvam habilidades manuais ou tomadas de decisão.

No contexto da Engenharia de Produção, a realização de atividades que envolvam a simulação de uma situação encontrada no mercado pelos alunos tem se mostrado bastante eficiente como estratégia de ensino-aprendizagem, visto que possibilita a contextualização do ambiente real, ampliando desta forma a percepção sobre o conhecimento adquirido em sala de aula (ZANOTTI; CARMO, 2011). Nesta mesma linha de raciocínio, de acordo com Satolo (2011), a utilização de simulação no processo de ensino na Engenharia de Produção apresenta diversos benefícios, dentre os quais destacam-se: a possibilidade de representar a complexidade de um sistema de produção de forma simples, a capacidade de mensurar os efeitos de diferentes cenários a partir de diferentes tomadas de decisão, a formação de competências que não são abrangidas plenamente pelo modelo tradicional de ensino como, por exemplo, o trabalho em equipe e a capacidade de criticar situações reais.

\section{MÉTODO}

O presente estudo consiste em uma pesquisa empírica descritiva, pois tem o objetivo de criar um modelo que descreva de forma adequada as relações causais que podem existir na realidade e leva a uma compreensão dos processos reais (MIGUEL, 2012). A abordagem utilizada foi a qualitativa, pois a preocupação principal do estudo foi obter informações sobre a perspectiva dos indivíduos, bem como interpretar o ambiente em que a problemática acontece. Esta abordagem implica no fato que o ambiente natural dos indivíduos é o ambiente da própria pesquisa, o que vai de acordo com as técnicas de pesquisa que foram utilizadas.

Em síntese, as metodologias utilizadas neste trabalho tiveram por finalidade viabilizar a criação, padronização e implementação de uma atividade lúdica que segue os princípios norteadores das metodologias ativas de aprendizagem. Esta atividade se divide em dois momentos principais, no primeiro é realizado a fabricação e venda de três tipos de produtos, conforme o sistema de produção empurrado e no segundo momento aplicam-se melhorias no processo antigo e efetua-se a fabricação e venda dos mesmos três tipos de produtos, porém conforme o sistema de produção puxado. Esses dois momentos têm por finalidade fazer com que o aluno "sinta na pele" a real diferença entre os modelos de produção, assim como os problemas enfrentados por cada um destes.

Para tanto, foram incluídas as seguintes etapas:

1) Estudar os conceitos operativos de educação em engenharia e aprendizagem ativa;

2) Elaborar a atividade lúdica;

3) Catalogar todas as etapas da atividade;

4) Descrever os materiais necessários para a aplicação da atividade;

5) Aplicar a atividade lúdica desenvolvida;

6) Elaborar o procedimento operacional padrão, com base na aplicação e nos feedbacks recebidos dos participantes.

\section{PROCESSO CRIATIVO DA ATIVIDADE LÚDICA}

A necessidade de se incluir métodos alternativos de ensino para os alunos da engenharia de produção, levou ao desenvolvimento de uma atividade lúdica acerca dos sistemas de produção. A atividade foi pensada de forma a simular uma fábrica que retratasse algumas das situações problemáticas que os alunos irão encontrar em um cenário real, como, por exemplo, linhas de produção desbalanceadas, excesso de estoque de matérias primas, produtos 
defeituosos, entre outras. Para representar tais situações foi desenvolvida uma linha de produção utilizando miçangas de diferentes cores dispostas em potes para montar os produtos, sendo que cada linha requer duas cores de miçangas.

A simulação se dividiu em dois momentos. No primeiro foi reproduzido um sistema empurrado de produção, onde os alunos foram organizados em linha e cada um foi responsável por colocar um tipo de miçanga na confecção dos produtos. A linha foi disposta de forma que pessoas que montavam o mesmo produto não ficassem próximas às outras, fazendo com que todos os itens passassem por todos operadores da linha, mesmo que alguns destes não fossem os responsáveis por determinado tipo. Além disso, um produto não podia ser passado adiante, caso o próximo operador estivesse ocupado. Isto acarretava o surgimento de filas de espera, operadores ociosos, excesso de estoque em processo e maior tempo de produção.

No segundo momento, o sistema foi mudado de empurrado para puxado, os alunos foram dispostos em células e os postos passaram a ser flexíveis, podendo produzir qualquer um dos produtos por completo; desta forma os problemas que ocorriam no primeiro momento foram solucionados. Em cada um dos cenários foi proposta uma condição de mercado: no sistema empurrado, o mercado, representado por um sorteio aleatório de dados, comprava os produtos apenas quando a fábrica terminava de produzir tudo, geralmente havendo falta de alguns produtos e excesso de outros. Já no sistema puxado, a fábrica só começava a produzir após serem realizados os pedidos do mercado, desta forma os produtos eram sempre feitos na quantidade exata em que foram solicitados.

\section{ELABORAÇÃO DO POP}

A elaboração de um Procedimento Operacional Padrão (POP) teve o intuito de tornar as tarefas e regras da atividade bem claras, tanto para os alunos quanto para o professor que será responsável pela aplicação, sem que haja a necessidade de um treinamento prévio para a realização da dinâmica. Para a produção do POP foi preciso aplicar a dinâmica em um grupo de controle, a fim de constatar as principais dificuldades encontradas pelos alunos e verificar se eles realmente entenderam o conteúdo abordado. A seguir foi pedido o feedback dos participantes que possibilitou identificar quais atividades eram essenciais e quais não.

Detectadas as falhas, a dinâmica foi reajustada e, para averiguar se houve melhoria no processo de aprendizagem, sua forma reestruturada foi aplicada em um outro grupo de controle. Finalmente, após a realização da atividade, o objetivo de fazer com que os alunos percebessem a diferença entre os sistemas de produção empurrado e puxado foram alcançados. Foi possível perceber melhorias em diversos pontos, como a redução do tempo de produção, redução de materiais em estoque, flexibilização da linha, entre outros. Além disso, o feedback dos alunos foi positivo tanto em relação à execução da atividade quanto ao conteúdo abordado.

Sendo assim, o POP ficou estruturado da seguinte forma:

- Descrição: Descreve de forma resumida a atividade a ser realizada.

- Objetivos: Aponta a finalidade da descrição do processo, o que ele pretende alcançar, melhorar, esclarecer, regular ou instruir.

- Pré-requisitos: Lista toda informação ou material necessário antes de executar o processo ou atividade.

- Materiais e recursos: Lista os materiais e a quantidade, caso necessários no processo.

- Descrição de tarefas: Provê a descrição das atividades necessárias para executar o processo. Cada atividade é descrita de maneira didática, com o maior nível de detalhamento possível. Define-se o tempo médio, necessário para conclusão da tarefa. 
Além disso, identifica o responsável pela sua execução bem como seu substituto eventual.

- Material de suporte e documentos relacionados: Lista e informa onde obter materiais que podem auxiliar na execução do procedimento, tais como: sites, links da internet para download de documentos, manuais, apostilas, outros POP's etc.

\section{CONCLUSÃO}

O ensino na engenharia tem demandado cada vez mais o uso de metodologias que contribuem para o desenvolvimento de novas competências e de novos valores. Desse modo, a utilização de técnicas de aprendizagem ativa é uma forma de capacitar os alunos para lidar com desafios, problemas, intervenções e proposições.

O presente trabalho resultou na criação e padronização de uma atividade lúdica que possibilita trabalhar com diversos conceitos relacionados à engenharia de produção ao simular um fábrica com linhas de produção puxada e empurrada, proporcionando assim a vivência de algumas das situações problemáticas que os alunos irão encontrar em um cenário real.

A elaboração do POP dessa atividade teve o intuito de tornar as tarefas e regras bem claras, possibilitando assim, que qualquer leitor interessado no assunto, juntamente com um grupo de pessoas, possa realizar a atividade sem que haja a necessidade de um treinamento prévio. Além disso, o POP garante que a atividade lúdica será realizada sempre da mesma forma, assegurando a eficácia durante a execução da tarefa e evitando desvios e variações ao longo dos processos. Ademais, facilita na organização, pois lista todos os materiais necessários e a forma correta de organizá-los.

A padronização por meio do POP permitirá que a atividade lúdica possa ser aplicada nos próximos anos por professores que não tenham aplicado a dinâmica anteriormente. Fazendo, assim, com que os esforços, recursos e o tempo necessários para a criação/elaboração dessa atividade não se percam ao longo do tempo, além de contribuir para que a atividade seja aplicada em sala mais vezes, visto que os professores provavelmente não teriam todo esse tempo de planejamento de aula para que pudessem elaborar uma atividade com esse porte.

Como sugestão para trabalhos futuros, recomenda-se a aplicação da atividade lúdica dentro da sala de aula como parte do planejamento didático pedagógico, pois desta forma será possível mensurar o impacto de tal atividade na formação técnica do engenheiro de produção.

\section{REFERÊNCIAS}

BARBOSA, Eduardo Fernandes; MOURA, Dácio Guimarães de. Metodologias ativas de aprendizagem no ensino de engenharia. In: International Conference On Engineering And Technology Education, 13., 2014, Portugal. Metodologias ativas de aprendizagem no ensino de engenharia [...]. [S. l.: s. n.], 2014. Disponível em:

http://copec.eu/intertech2014/proc/works/25.pdf. Acesso em: 3 jul. 2020.

CAMPOS, Vicente Falconi. Qualidade Total: Padronização de empresas. 2. ed. Nova Lima: Editora FALCONI, 2014.

COBENGE. Repositório GTAAEE. In: Artigos relacionados à Aprendizagem Ativa na Educação em Engenharia (AAEE). [S. l.], 2019. Disponível em: https://sites.google.com/site/gtaaeecobengea/. Acesso em: 12 jul. 2020. 
COSTA, Wilma da Cruz, PINHO, Kátia Elisa Prus. A Importância e a Contribuição do Lúdico no Processo Educacional. 2012. Disponivel em:

$\langle$ http://www.diaadiaeducacao.pr.gov.br/portals/pde/arquivos/1681-8.pdf $>$. Acesso em 14 de Mai. 2020.

FARIAS, Pablo Antonio Maia de; MARTIN, Ana Luiza de Aguiar Rocha; CRISTO, Cinthia Sampaio. Aprendizagem Ativa na Educação em Saúde: Percurso Histórico e Aplicações.

Revista brasileira de educação médica, [S. 1.], v. 39, n. 1, 2015. Disponível em: Rev. bras. educ. med. vol.39 no.1 Rio de Janeiro Jan./Mar. 2015. Acesso em: 15 abr. 2020.

HEINIG, Otilia Lizete de Oliveira Martins; SCHLICHTING, Thais de Souza Schlichting. Aprendizagem Ativa na Engenharia: um Enfoque nas Práticas de Linguagem. Revista Eletrônica Engenharia Viva. MG, Vol. 2 No. 1, 2015. Disponível em <https://www.revistas.ufg.br/ijaeedu/article/view/33283>. Acesso em 03 de julho de 2020.

LIMA, Luan Eloy; SOUZA, Flávia Roberta Ferreira. Gerenciamento de Procedimentos Operacionais através de Sistema Informativo: Estudo de Caso da KB Cervejarias. Revista de Administração, Sociedade e Inovação, v. 1, n. 1, p. 37-52, 2015.

LIMBERGER, Jane Beatriz. Metodologias ativas de ensino-aprendizagem para educação farmacêutica: um relato de experiência. Interface - Comunicação, Saúde, Educação, [S. 1.], v. 17, n. 47, oct/dec 2013. Disponível em: https://doi.org/10.1590/1807-57622013.3683. Acesso em: 15 abr. 2020.

MASSA, Monica de Souza. Ludicidade: da Etimologia da Palavra à Complexidade do Conceito. Aprender - Caderno de Filosofia e Psicologia da Educação, [S.1.], n. 15, dez. 2017. ISSN 2359-246X. Disponível em: <http://periodicos2.uesb.br/index.php/aprender/article/view/2460>. Acesso em: 13 jul. 2020.

MEC. CNE. Parecer Homologado Despacho do Ministro, publicado no D.O.U. de 23/4/2019, Seção 1, Pág. 109. Disponível em: $<$ http://portal.mec.gov.br/index.php?option=com docman\&view=download\&alias=109871pces001-19-1\&category_slug=marco-2019-pdf\&Itemid=30192>. Acesso em 30 de Abr. de 2020.

MIGUEL, Paulo Augusto Cauchick (Coord.). Metodologia de pesquisa em engenharia de produção e gestão de operações. 2. ed. Rio de Janeiro: Elsevier, Associação Brasileira de Engenharia de Produção, 2012. xviii.

PAZIN FILHO, Antonio; SCARPELINI, Sandro. Simulação: Definição. Medicina, Ribeirão Preto - MG, v. 40, n. 2, p. 162-166, abr/jun 2007. DOI https://doi.org/10.11606/issn.21767262.v40i2p162-166. Disponível em: https://www.revistas.usp.br/rmrp/article/view/312. Acesso em: 15 abr. 2020.

PRINCE, Michael. Does Active Learning Work? A Review of the Research. Wiley Online Library. RJ. 2004. Disponível em < https://onlinelibrary.wiley.com/doi/abs/10.1002/j.21689830.2004.tb00809.x?ca 
sa token=gwlqlREtwhsAAAAA\%3A1ttQisd-

tGS3i3Xc4NoTUBQxzgtBR_7gKS_HOAOI8r7HCzCpjPnAq2w3yqBZIvVV7b7gJZ_ExcM01 Ap9s>. Acesso em 12 jul. 2020.

RESENDE, Laura Amâncio; SILVA, Humberto Felipe; PRADO, Cíntia Fernanda do; NETO, Morun Bernardino. Gestão e Tecnologia em Educação. In: RESENDE, Laura Amâncio. Análise da Percepção de Graduandos em Engenharia Quanto à Importância de Metodologias de Aprendizagem Ativa. Belo Horizonte: Poisson, 2018. v. 1. Disponível em: https://www.poisson.com.br/livros/gte/volume1/GTE volume1.pdf\#page=42. Acesso em: 3 jul. 2020.

RIBEIRO NETO, João Batista M; TAVARES, José da Cunha; HOFFMANN, Silvana Carvalho. Sistema de gestão integrados: qualidade, meio ambiente, responsabilidade social, segurança e saúde no trabalho. 4. ed. São Paulo: Editora Senac, 2013.

RIZZETTI, D. M.; CUNHA, D. E.; MOURA, G. L. DE; SCHLOSSER, A. L. DA C. Padronização de Processos e Rotinas no Núcleo de Controle e Manutenção do Sistema de Pagamento. TPA - Teoria e Prática em Administração, v. 5, n. 1, p. 239-260, 30 jun. 2015.

SATOLO, Eduardo Guilherme. Modelo de Simulação Aplicado ao Conceito da Produção Enxuta no Ensino de Engenharia de Produção. Revista Gestão Industrial, Paraná, v. 07, n. 02, p. 201-216, 2011. Disponível em: https://periodicos.utfpr.edu.br/revistagi/issue/view/71. Acesso em: 15 abr. 2020.

SOUZA, Samir Cristino de; DOURADO, Luis. Aprendizagem Baseada Em Problemas (Abp): Um Método De Aprendizagem Inovador Para O Ensino Educativo. HOLOS, [S.1.], v. 5, p. 182-200, out. 2015. ISSN 1807-1600. Disponível em:

<http://www2.ifrn.edu.br/ojs/index.php/HOLOS/article/view/2880>. Acesso em: 12 jul. 2020. doi:https://doi.org/10.15628/holos.2015.2880.

VILLAS-BOAS, Valquíria; MATTASOGLIO NETO, O. Aprendizagem ativa na educação em engenharia. In: Congresso Brasileiro de Educação em Engenharia, - COBENGE Blumenau. 2011. Disponível em < https://edisciplinas.usp.br/pluginfile.php/4935407/mod_resource/content/1/Capitulo\% 20SD3\%20Aprendizagem\%20Ativa VERSAO FINAL.pdf.>Acesso em: 13 mai. 2020.

ZANOTTI, Juliane Silvia; CARMO, Cintia Tavares do. Simulação Como Estratégia de Ensino-Aprendizagem: Estudo de Fatores Contextuais Críticos Para o Sucesso em Sala de Aula. In: COBENGE, 39., 2011, Blumenau - SC. Anais [...]. Blumenau - SC: [s. n.], 2011. Tema: Educação em Engenharia, Disponível em: http://www.abenge.org.br/cobenge/interna.php?ss=8\&ctd=86. Acesso em: 15 abr. 2020. 


\title{
DEVELOPMENT AND STANDARDIZATION OF A PLAYFUL TEACHING ACTIVITY OF PRODUCTION SYSTEMS CONCEPTS
}

\begin{abstract}
This work consists in development and standardization of a playful teaching activity which follows the well-known principles of active learning, with the main objective of contributing to the improvement of teaching concepts for production systems in the Production Engineering course for a higher educational institution. Moreover, it allows students to develop skills to deal with and solve problems found throughout their professional careers. The work is classified as a descriptive empirical research and by using results already obtained so far, we concluded that the development of the activity through the use of the standardoperating procedures (SOPS) is fundamental because it ensures that the activity will be carried out correctly. In addition, it ensures the effectiveness of the teaching methods used, facilitates, and streamlines the preparation of classes by the teacher and, consequently, saves planning time.
\end{abstract}

Keywords: Engineering education. Active learning. Standardization of activities. 